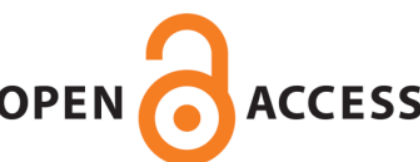

UWS Academic Portal

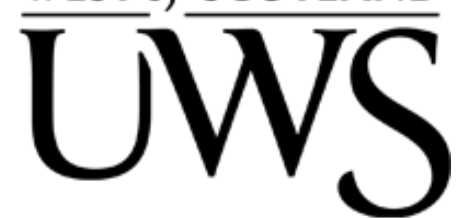

\title{
Pattern reconfigurable antenna with multi-port excitation for K-band application
}

Chilukuri, Sulakshana; Dahal, Keshav; Lokam, Anjaneyulu

Published in:

IEEE International Conference on Microwaves, Antennas, Communications and Electronic Systems (COMCAS), 2017

DOI:

10.1109/COMCAS.2017.8244751

Published: 04/01/2018

Document Version

Peer reviewed version

Link to publication on the UWS Academic Portal

Citation for published version (APA):

Chilukuri, S., Dahal, K., \& Lokam, A. (2018). Pattern reconfigurable antenna with multi-port excitation for K-band application. In IEEE International Conference on Microwaves, Antennas, Communications and Electronic Systems (COMCAS), 2017 (pp. 1-5). IEEE. https://doi.org/10.1109/COMCAS.2017.8244751

\section{General rights}

Copyright and moral rights for the publications made accessible in the UWS Academic Portal are retained by the authors and/or other copyright owners and it is a condition of accessing publications that users recognise and abide by the legal requirements associated with these rights. 


\title{
Pattern reconfigurable antenna with multi-port excitation for K-band application
}

\author{
Ch. Sulakshana, Keshav Dahal, and L. Anjaneyulu \\ ${ }^{1,2}$ AVCN Research Center, University of West of Scotland, Paisley, PA12BE, UK. ${ }^{3}$ Department of \\ Electronics and Communication Engineering, National Institute of Technology, Warangal, 506004, \\ India
}

\begin{abstract}
A compact coplanar waveguide (CPW) fed reconfigurable antenna with pattern diversity using multi-port excitation is designed. The basic antenna consists of a rectangular patch of size $5 \mathrm{mmx} 4 \mathrm{~mm}$ which is fed by four ports independently. By exciting the patch with each individual port, the direction of the radiation pattern changes by $90^{\circ}$. With the use of CPW feed technique, a very wide impedance bandwidth of around $9 \mathrm{GHz}$ covering the whole $18 \mathrm{GHz}-27 \mathrm{GHz}$ (K-band) is achieved. The proposed antenna can be used for different satellite communication applications like earth exploration, radio navigation and location, mobile satellite communications which comes under K-band. The measured return loss and pattern characteristic results are in good agreement with simulated ones.
\end{abstract}

Index Terms - Coplanar waveguide, correlation coefficient, impedance bandwidth, pattern diversity, reconfigurable antennas, return loss, satellite communications.

\section{INTRODUCTION}

The concept of reconfigurable antennas was born to satisfy the increasing demands of wireless environment. The $\mathrm{RF}$ terminals used in such wireless communication systems must have multi-functional properties that adapt to system requirements such as frequency diversity, polarization diversity and radiation pattern selectivity. Reconfigurability sometimes makes the system intelligent. Smart systems such as Software Defined Radio (SDR), Cognitive Radio, adaptive multiple-input multiple-output communication systems use different reconfiguration schemes to adapt to changing system requirements like physical link conditions which improves the system performance by providing the robustness to varying channel conditions. These antennas provide cost effective solutions by incorporating multiple elements in a single physical device which reduces the total number of components used, thereby reducing the overall size of the system. The pattern reconfigurable antennas have the ability to dynamically change their radiation properties that adapt to the wireless channel characteristics. In a rich scattered wireless environment, an antenna that directs the main beam with increased gain to the desired coverage area while suppressing the unwanted beam in other directions, is required. This type of antennas mitigate the multi-path interference and allows higher rate of data transmission in a given band that enhances the spectral efficiency and improves the communication link performance because of the adaptive patterns it provides.

Many pattern diversity antennas have been designed in the past and many more are being done recently. A high gain beam switching antenna that can steer the beam to five different directions using four parasitic elements and one main radiating element for WiMAX applications is proposed [1]. Likewise, two parasitic elements and four switches are incorporated in UWB monopole antenna to get pattern diversity [2]. A new technique in which a parasitic structure (wire, loop or any shape) is rotated around a monopole to get rotated patterns [3]. An electro-active polymer actuator is used for rotation with a triangular parasitic element to get different beams. A CPW to slot line feed transition method is proposed to get pattern diversity [4]. Three different patterns are obtained with three feed modes; CPW, left slot line and right slot line. Dual-band reconfigurable frequencyselective reflectors (RFSR) are designed and applied to form a right-angle corner reflector antenna with reconfigurable patterns [5]. The concept of Yagi-Uda antenna is implemented on a planar patch to achieve beam steering by manipulating the status of the parasitic patches through switching mechanism to act as reflectors or directors [6]. Active Frequency Selective Surfaces (AFSS) are used to build a $360^{\circ}$ beam steerable antenna with not only single and multi-beam configurations but also proportional beams are realizable with this continuous tuning capability [7].

The main aim of this paper is to achieve pattern diversity by using geometrically symmetrical shaped antenna with small size and less complexity. Very less literature is found on wide band pattern diversity antennas. Hence, we propose a very simple, compact and wide band pattern diversity antenna with four port excitations where the pattern changes its direction by $90^{\circ}$ with each individual port exciting the main radiating patch. A coplanar waveguide (CPW) feed is used to excite the antenna. So far, pattern diversity antennas are designed with conventional microstrip feeding structures but this paper proposes CPW feed because of its many advantages such as; reduction in radiation loss, improved bandwidth, easy surface mounting of active and passive devices, reduced cross talk effects between adjacent lines because of ground plane between any two adjacent lines, simplified fabrication [8]. The configuration of the proposed antenna is briefly discussed in Section II and the simulated and measured results are presented in Section III and Section IV. The practical results are in good agreement with the simulated ones. Finally, the conclusions and useful applications are presented in Section V. 


\section{ANTENNA CONFIGURATION}

The geometry of the proposed antenna is shown in Fig. 1. The antenna is fabricated on a $1.6 \mathrm{~mm}$ thick low cost FR4 substrate whose dielectric constant is 4.4 with loss tangent 0.02 . The length and width of basic rectangular patch are $5 \mathrm{~mm}$ and $4 \mathrm{~mm}$ respectively. The area of the antenna is $14 \mathrm{~mm} \times 13 \mathrm{~mm}$ X $1.6 \mathrm{~mm}$ which is very compact in size.

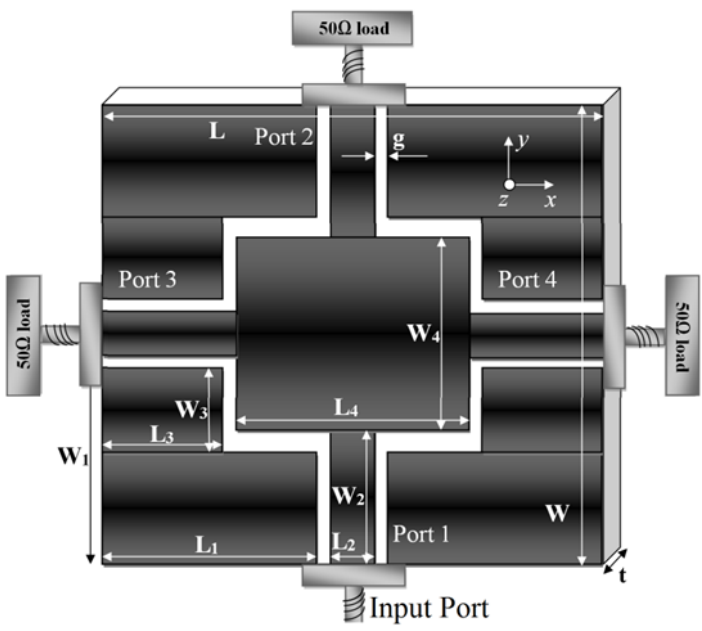

Fig. 1. Geometry of the proposed pattern diversity antenna. The parameters are: $\mathrm{L}=14 \mathrm{~mm}, \mathrm{~W}=13 \mathrm{~mm}, \mathrm{t}=1.6 \mathrm{~mm}, \mathrm{~g}=0.3 \mathrm{~mm}$, $\mathrm{L}_{1}=6.05 \mathrm{~mm}, \mathrm{~W}_{1}=5.5 \mathrm{~mm}, \mathrm{~L}_{2}=1.3 \mathrm{~mm}, \mathrm{~W}_{2}=4.5 \mathrm{~mm}, \mathrm{~L}_{3}=4 \mathrm{~mm}$, $\mathrm{W}_{3}=1.55 \mathrm{~mm}, \mathrm{X}_{1}=5 \mathrm{~mm}, \mathrm{Y}_{1}=4 \mathrm{~mm}$.

The signal is fed through the four ports, independently, which are placed at the four sides of the basic rectangular patch. When the patch is fed by one port, all the remaining ports are terminated by a $50 \Omega$ load for proper impedance match. The signal fed through each individual port changes the spatial current distribution on the radiating patch and thus changes the radiation pattern.

\section{SimULATION RESULTS AND DisCUSSIONS}

The simulations are carried out in Ansoft HFSS software package which is based on Finite Element Method. Fig. 2 shows the reflection coefficients of the antenna fed by each port individually. The operating frequency of the antenna when it is fed through Port 1 is $22.4 \mathrm{GHz}$ with $-10 \mathrm{~dB}$ impedance bandwidth (BW) of $10.9 \mathrm{GHz}(48.8 \%)$, through Port 2 it is $22.8 \mathrm{GHz}$ with a BW of $10.83 \mathrm{GHz}(47.35 \%)$, through Port 3 it is $22.3 \mathrm{GHz}$ with a BW of $8.02 \mathrm{GHz}$ $(35.98 \%)$ and through Port 4 it is $22.17 \mathrm{GHz}$ with a BW of $9.99 \mathrm{GHz}(45.06 \%)$. The radiation characteristics of the basic rectangular patch are determined by the electric or magnetic current distribution on the radiating structure which in turn are responsible for changing frequency characteristics. Because of this relationship between the source currents and the resulting radiation, it is difficult to achieve pattern reconfigurability without having much changes in frequency characteristics. Hence there is a slight change in frequency in each operating mode.
Fig. 3 shows the 3D plots of the radiation patterns of the antenna operating in four different modes. It is observed that when the antenna feed is changed from Port 1 to Port 3, Port 3 to Port 2, Port 2 to Port 4 and again to Port 1, the radiation pattern changes by $90^{\circ}$ each time. There is a change in the area covered by the minor lobe shown in Fig. 3(a) and Fig. 3(c), because of the difference in the dimensions of the length and width of the radiating patch. Hence, the radiation intensity of the main lobe in Fig. 3(c) is less when compared to Fig. 3(a) which in turn increases the back lobe radiation. It is observed from Fig. 2 and Fig. 3 that frequency characteristics are maintained while changing the radiating characteristics when the antenna is fed through different ports individually which satisfies the principle of pattern reconfigurability.

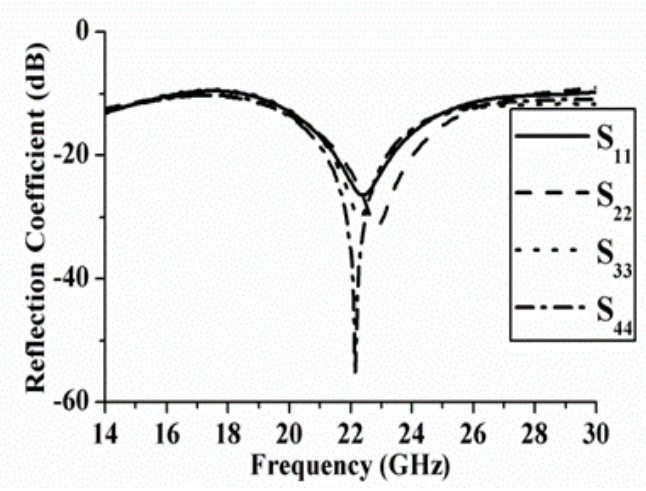

Fig. 2. Reflection coefficients of proposed pattern diversity antenna.

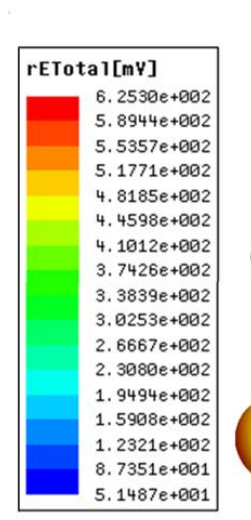

(a) Port 1

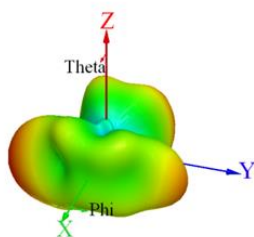

(c) Port 3

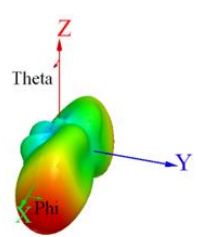

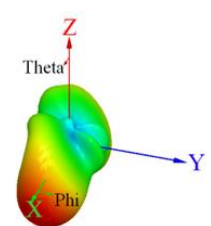

(b) Port 2

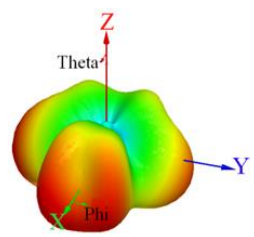

(d) Port 4
Fig. 3. 3D radiation pattern of the proposed pattern diversity antenna at (a) $22.4 \mathrm{GHz}$ (b) $22.87 \mathrm{GHz}$ (c) $22.29 \mathrm{GHz}$ (d) $22.17 \mathrm{GHz}$.

The transmission coefficients of the antenna when fed through Port 2 are shown in Fig. 4. It is known that $S_{i j}=S_{j i}$ and $\mathrm{S}_{12}=\mathrm{S}_{34}$ because of the equal lengths of the opposite sides of the rectangle. Also, $\mathrm{S}_{31}=\mathrm{S}_{41}=\mathrm{S}_{32}=\mathrm{S}_{42}$. The average correlation between the total power radiated by antenna when fed through different ports within the threedimensional space, called envelope correlation coefficient, (ECC) is calculated to evaluate the diversity performance [9]. 


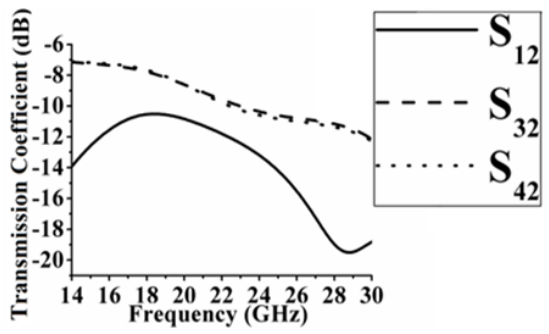

Fig. 4. Transmission coefficients of the proposed pattern diversity antenna when it is fed through Port 2.

The correlation between the ports 1 and 2 is given by (1) which is calculated from S-parameters obtained in the simulations. A good diversity performing antenna has the correlation factor $<0.5$. It is known that, $\rho_{i j}=\rho_{j i}$, and in our case $\rho_{13}=\rho_{14}$, because the equal length of the two vertical sides of the rectangle. Fig. 5 shows the ECCs obtained from the simulated S-parameters.

$$
\rho_{12}=\frac{\left|\mathrm{S}_{11}{ }^{*} \mathrm{~S}_{12}+\mathrm{S}_{21}{ }^{*} \mathrm{~S}_{22}\right|^{2}}{\left(1-\left(\left|\mathrm{S}_{11}\right|^{2}+\left|\mathrm{S}_{21}\right|^{2}\right)\right)\left(1-\left(\left|\mathrm{S}_{22}\right|^{2}+\left|\mathrm{S}_{12}\right|^{2}\right)\right)}
$$

The ECC for $\rho_{12}$ is $<0.12$ and for $\rho_{13}$ is $<0.04$ which satisfies the low correlation criteria of ECC $<0.5$.

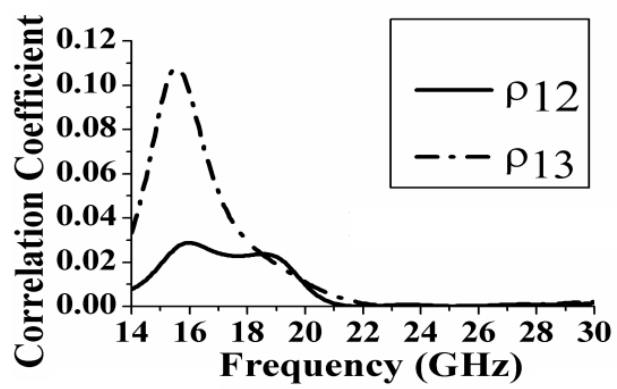

Fig. 5. Simulated ECs of the proposed antenna.

A parametric study has been carried out to optimize certain geometric parameters of the proposed antenna such as gap ' $\mathrm{g}$ ' $(0.1 \mathrm{~mm}$ to $0.3 \mathrm{~mm})$ between the central conductor of the CPW feed and the ground, the thickness ' $h$ ' $(0.5 \mathrm{~mm}$ to $3.2 \mathrm{~mm}$ ) of the substrate and the length ' $\mathrm{X}_{1}{ }^{\prime}$ (3.5mm to $\left.5 \mathrm{~mm}\right)$ and width ' $Y_{1}$ ' (2mm to $4 \mathrm{~mm}$ ) of the rectangular patch. The $\mathrm{S}$-parameter curves of the proposed antenna with varying $\mathrm{X}_{1}, \mathrm{Y}_{1}$ and $\mathrm{g}$ for a constant thickness $(\mathrm{h}=1.2 \mathrm{~mm})$ are shown in Fig. 6 and the same with varying $h, g$ for a constant $X_{1}=5 \mathrm{~mm}$ and $Y_{1}=4 \mathrm{~mm}$ are shown in Fig. 7 . It is observed from the Fig. 7 that, as the thickness of the substrate is increased from $0.5 \mathrm{~mm}$ to $1.6 \mathrm{~mm}$, the operating frequency decreases and again it increases from $h=2.4 \mathrm{~mm}$. It can be concluded from both the figures that as the gap spacing between the CPW feed line and ground is increased, the resonant frequency of the proposed antenna is shifted towards left (decreased) side of the origin. The same is observed when the length and width of the rectangle is increased.

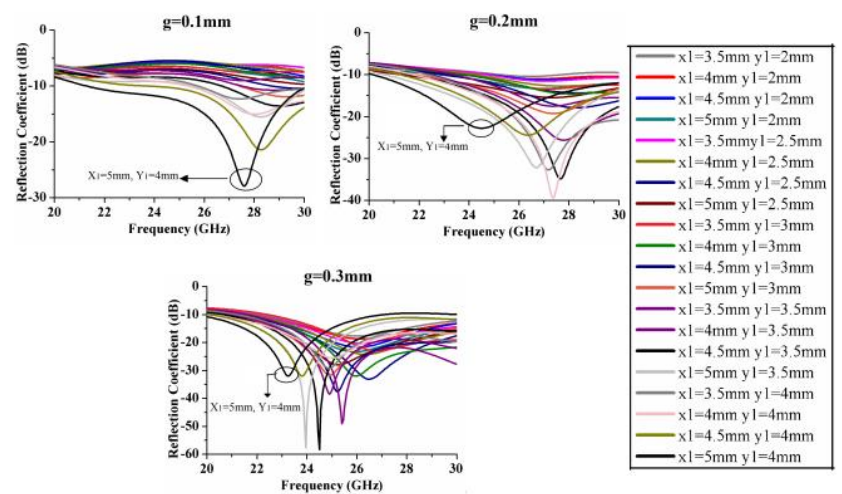

Fig. 6. S-Parameter curves for constant $\mathrm{h}=1.2 \mathrm{~mm}$ and varying $g, X_{1}, Y_{1}$.
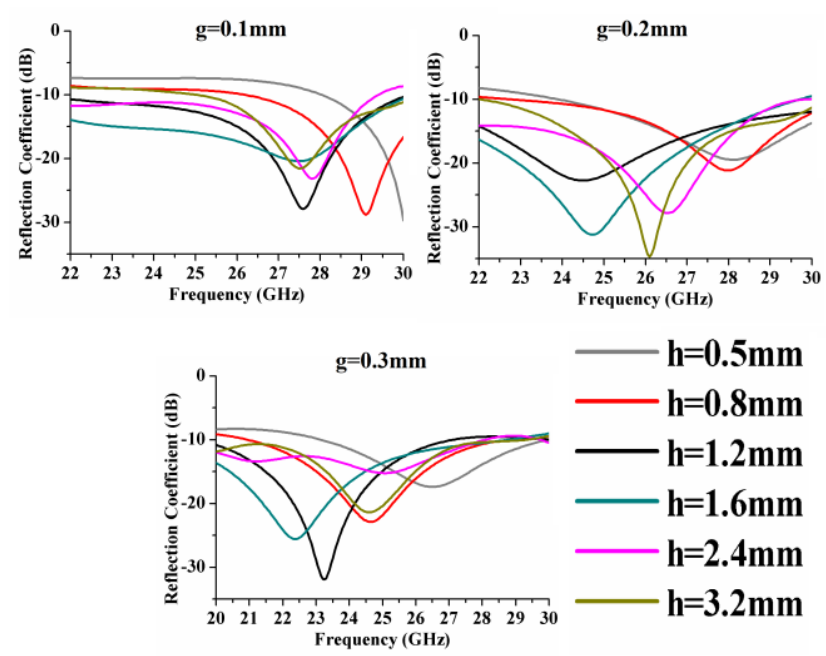

Fig. 7. S-Parameter curves for constant $X_{1}=5 \mathrm{~mm}, Y_{1}=4 \mathrm{~mm}$ and varying $g$ and $h$.

\section{MEASURED RESULTS}

The proposed reconfigurable antenna was fabricated on a low cost FR4 substrate with dielectric constant 4.4 and thickness $1.6 \mathrm{~mm}$ and tested to validate the simulated results. The photograph of the prototype antenna used for the measurement is shown in Fig. 8. All the return loss measurements were taken using Agilent E8363C PNA Series Microwave Network Analyzer, which operates in the frequency range $10 \mathrm{MHz}$ to $40 \mathrm{GHz}$.

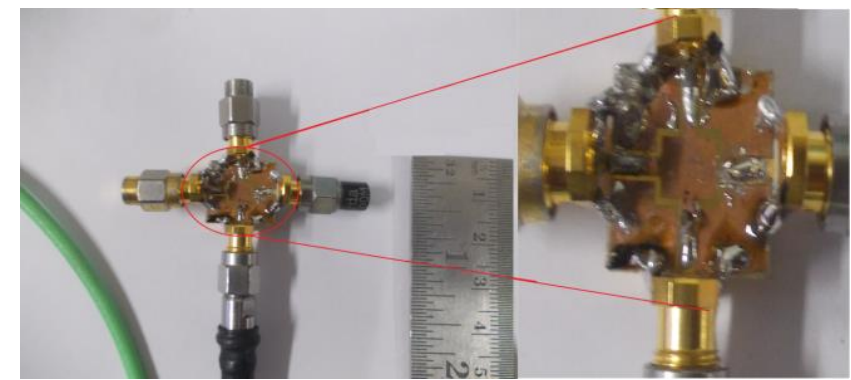

Fig. 8. Fabricated prototype of the proposed antenna with pattern diversity 
The simulated and measured reflection coefficients $S_{11}$ of the proposed reconfigurable antenna for all the operating modes are shown in Fig. 9. The reasons for the difference between simulated and measured results are due to minor fabrication inaccuracies, dielectric imperfections, and SMA connector solder losses (i.e., while testing, SMA connector can be treated as the extension of the ground which creates some impedance mismatch at the CPW feed). The measured and simulated results are tabulated in Table 1.
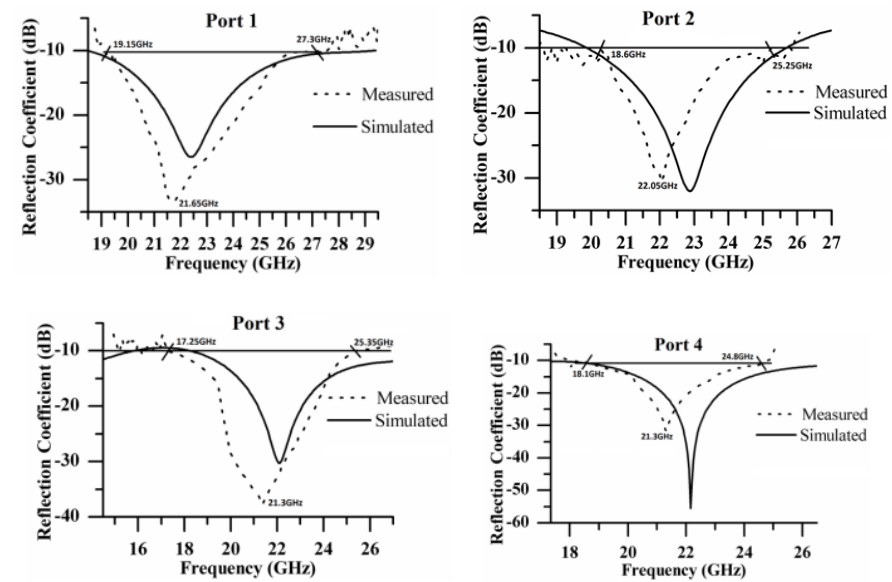

Fig. 9. Simulated and Measured Reflection coefficients of proposed reconfigurable antenna when fed through different ports.

The simulated and measured 2D polar cuts in azimuth and elevation planes depicting both co-polarization and crosspolarization for each port are shown in Fig. 10 and Fig. 11.
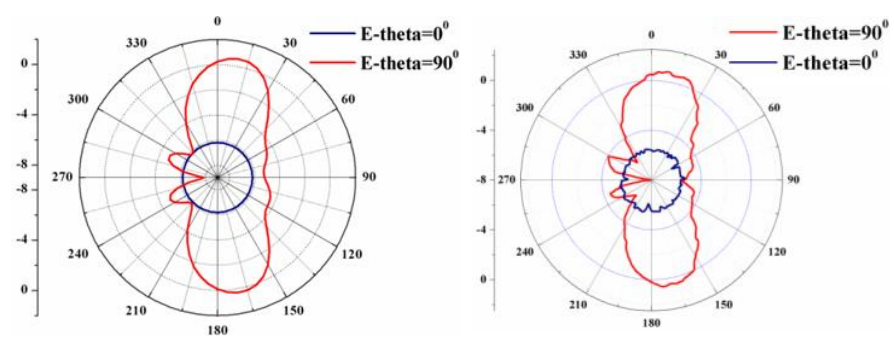

(a) Port 1
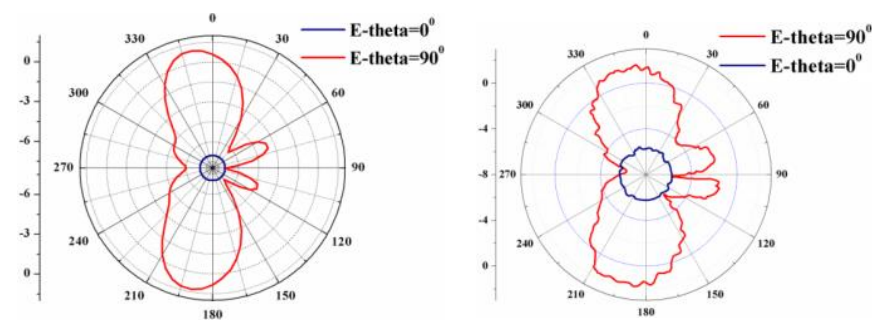

(b) Port 2
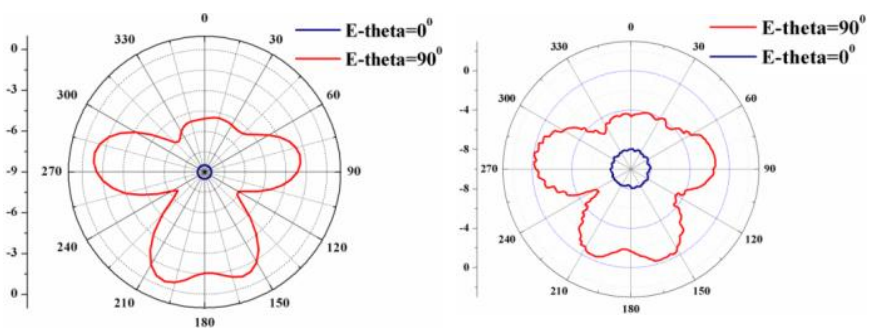

(c) Port 3
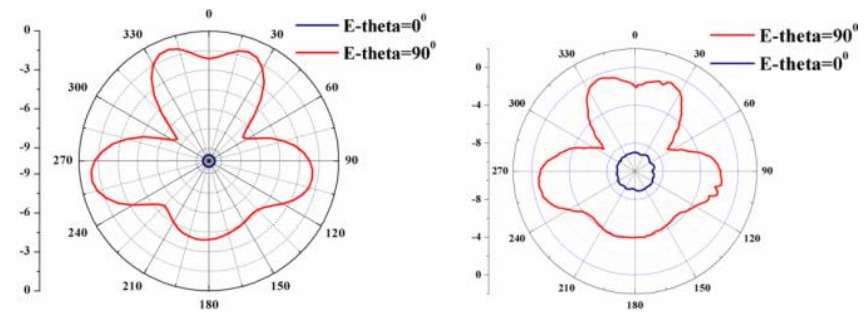

(d) Port 4

Fig 10. Simulated (Left side) Measured (Right side) 2D Radiation patterns of the pattern reconfigurable antenna in Elevation plane with excitations at different ports (a) $21.6 \mathrm{GHz}$ (b) $22.0 \mathrm{GHz}$ (c) $21.4 \mathrm{GHz}$ (d) $21.2 \mathrm{GHz}$

There is a change in the area covered by the minor lobe shown in Fig. 10(a) and Fig. 10(c), because of the difference in the dimensions of the length and width of the radiating patch. Hence, the radiation intensity of the main lobe in Fig. 10 (c) is less when compared to Fig. 10(a) which in turn increases the back lobe radiation.
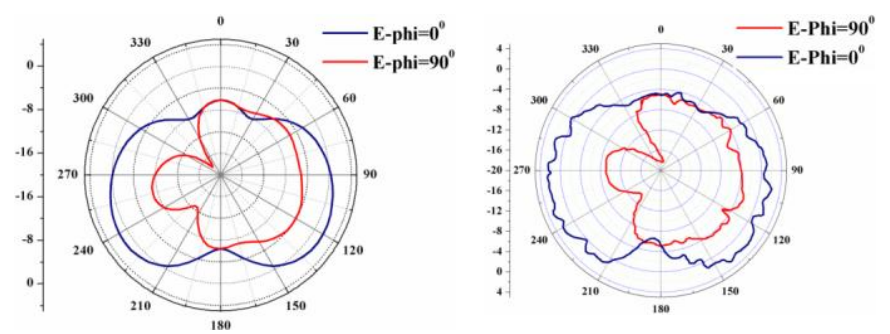

(a) Port 1
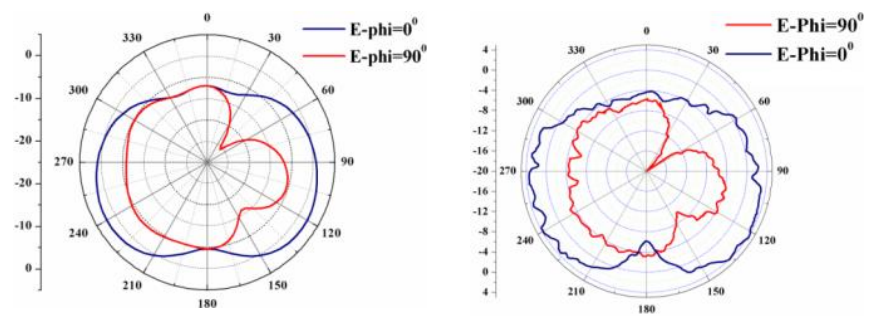

(b) Port 2 

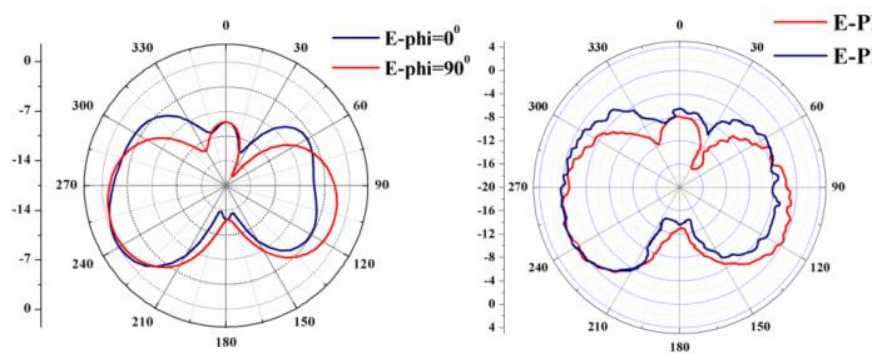

(c) Port 3

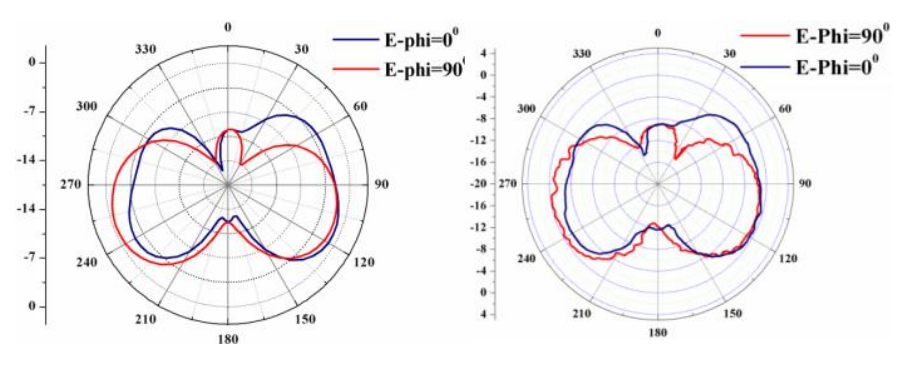

(d) Port 4

Fig. 11. Simulated (Left side) and Measured (Right side) 2D Radiation patterns of the pattern reconfigurable antenna in Azimuth plane with excitations at different ports (a) $21.6 \mathrm{GHz}$ (b) $22.0 \mathrm{GHz}$ (c) $21.4 \mathrm{GHz}$ (d) $21.2 \mathrm{GHz}$

It is observed from Fig. 9 that frequency characteristics are maintained while changing the radiating characteristics when the antenna is fed through different ports individually which satisfies the principle of pattern reconfigurability.

\section{CONCLUSION}

A very simple and compact pattern reconfigurable antenna using CPW feed is designed using four ports and analysed in this paper. A very wide impedance bandwidth is achieved by the use of CPW feeding technique. The antenna is capable of rotating its radiation pattern by $90^{\circ}$ with each port excitation without having major changes in frequency characteristics. A very good return loss and very low ECC is obtained between four ports which can be used in different MIMO applications. Due to its compact dimension and high isolation between the different ports and wide bandwidth, the proposed antenna is suitable for mobile satellite communication applications operated under K-band.

\section{ACKNOWLEDGEMENT}

The authors wish to acknowledge the funding support provided by EU-Erasmus Mundus SmartLink under Grant agreement (552077-EM-1-2014-1-UK-ERA) to carry out this research at the University of the West of Scotland, UK.

\section{REFERENCES}

[1] Jusoh, M., Sabapathy, T., Jamlos, M.F. \& Kamarudin, M.R, "Reconfigurable Four-Parasitic-Elements Patch Antenna for High-Gain Beam Switching Application," IEEE Antennas and Wireless Propagation Letters, vol. 13, pp. 79-82, 2014.

[2] Aboufoul, T., Parini, C., Xiaodong Chen \& Alomainy, A, "Pattern-Reconfigurable Planar Circular Ultra-Wideband Monopole Antenna," IEEE Transactions on Antennas and Propagation, vol. 61, no. 10, pp. 4973-4980, 2013.

[3] Jalali Mazlouman, S., Mahanfar, A, Soleimani, M., Chan, H., Menon, C \& Vaughan, R.G, "Pattern Reconfiguration by Rotating Parasitic Structure Using Electro-Active Polymer (EAP) Actuator," IEEE Transactions on Antennas and Propagation, vol. 62, no. 3, pp. 1046-1055, 2014.

[4] Yue Li, Zhijun Zhang, Jianfeng Zheng, Zhenghe Feng \& Iskander, M.F, "Experimental Analysis of a Wideband Pattern Diversity Antenna With Compact Reconfigurable CPW-toSlotline Transition Feed," IEEE Transactions on Antennas and Propagation, vol.59, no. 11, pp. 4222-4228, 2011.

[5] Chih-Hsiang Ko, I-Young Tarn \& Shyh-Jong Chung, "A Compact Dual-Band Pattern Diversity Antenna by Dual-Band Reconfigurable Frequency-Selective Reflectors With a Minimum Number of Switches," IEEE Transactions on Antennas and Propagation, vol. 61, no. 2, pp. 646-654, 2013.

[6] Jusoh, M., Aboufoul, T., Sabapathy, T., Alomainy, A. \& Kamarudin, M.R, "Pattern-Reconfigurable Microstrip Patch Antenna with Multidirectional Beam for WiMAX Application," IEEE Antennas and Wireless Propagation Letters, vol. 13, pp. 860-863, 2014.

[7] Liang Zhang, Qun Wu \& Denidni, T.A, "Electronically Radiation Pattern Steerable Antennas Using Active Frequency Selective Surfaces," IEEE Transactions Antennas and Propagation, vol. 61, no. 12, pp. 6000-6007, 2013.

[8] R.N. Simons, Coplanar Waveguide circuits, Components and systems. New York: J. Wiley \& Sons, 2001.

[9] Blanch, S., Romeu, J. \& Corbella, I, "Exact representation of antenna system diversity performance from input parameter description," Electronics Letters, vol. 39, no. 9, pp. 705-707, 2003.

TABLE I

MEASURED AND SIMULATED RETURN LOSS GRAPH PARAMETERS FOR PATTERN DIVERSITY ANTENNA

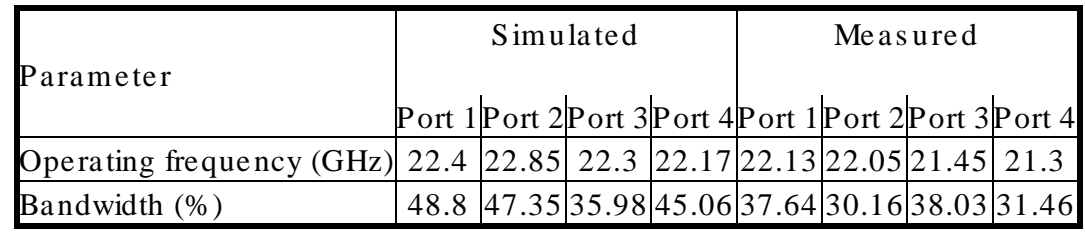

